\title{
A Practical Method to Prevent Cross-Infection When Performing Dermoscopy
}

\author{
Mohammed I. AlJasser ${ }^{1,2}$
}

1 Division of Dermatology, King Saud bin Abdulaziz University for Health Sciences, Riyadh, Saudi Arabia

2 King Abdulla International Medical Research Center, Riyadh, Saudi Arabia

Key words: pearl, dermoscopy, dermatoscopy, plastic wrap, rubber band, tie, wire, cross-infection, COVID-19

Citation: AlJasser MI. A practical method to prevnt cross-infection when performing dermoscopy. Dermatol Pract Concept. 2021;11(1):e2021110. DOI: https://doi.org/10.5826/dpc.1101a110

Accepted: June 22, 2020; Published: January 29, 2021

Copyright: @2021 AlJasser. This is an open-access article distributed under the terms of the Creative Commons Attribution License BY-NC-4.0, which permits unrestricted noncommercial use, distribution, and reproduction in any medium, provided the original author and source are credited.

Funding: None.

Competing interests: The author has no conflicts of interest to disclose.

Authorship: The author takes responsibility for this publication.

Corresponding author: Mohammed I. AlJasser MBBS FRCPC, Division of Dermatology, King Saud bin Abdulaziz University for Health Sciences. P.O. Box 3660, Riyadh 11481, Saudi Arabia. Email: mj_derma@hotmail.com

Dermoscopy is a valuable noninvasive diagnostic tool in dermatology. Contact dermoscopy is commonly used. Due to the potential risk of cross-infection by dermoscopy, several methods have been described to decrease this risk [1]. Commercially available disposable plastic covers are excellent for this purpose. However, cost and availability are limiting factors. Plastic wrap has been shown to be efficient in preventing transmission of viruses [2]. One disadvantage of this method is the limited flexibility of use. This is especially true when examining multiple body sites where the plastic sheet has to be moved to the next body site.

This issue can be resolved by using a small piece of plastic wrap and a rubber band (Figure 1). The plastic wrap is firmly stretched over the dermatoscope faceplate then fixed in place with the rubber band (Figure 2). Alternatively, a metal cable tie can be used (Figure 3). After completing the examination, this customized cover can be easily removed and discarded.

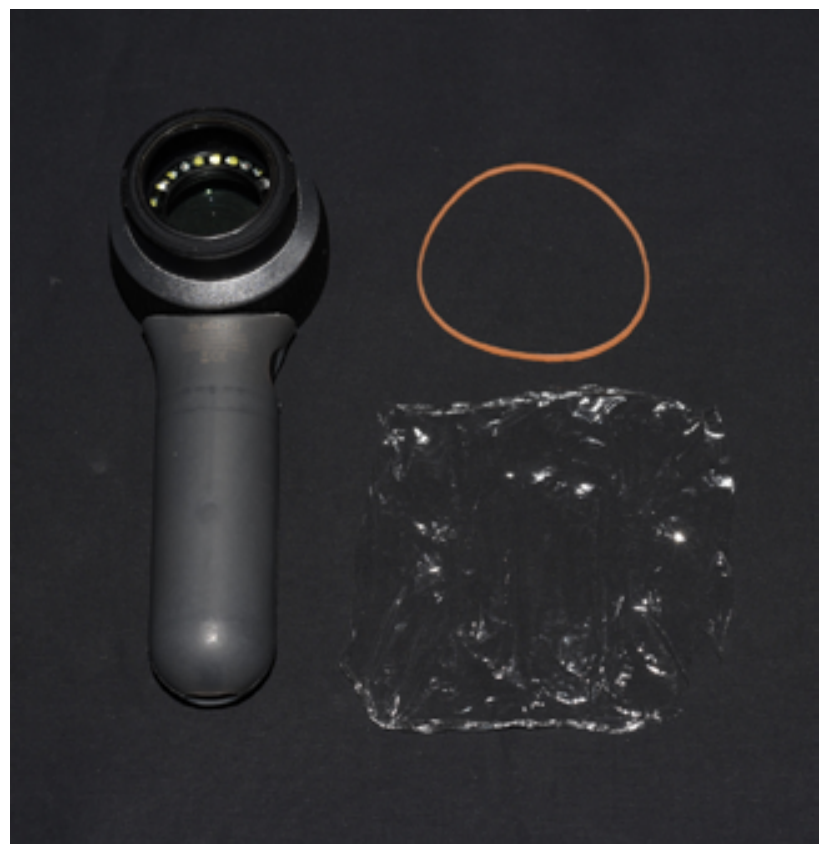

Figure 1. A small piece of plastic wrap and rubber band is required to create a custom dermoscopy faceplate cover. 


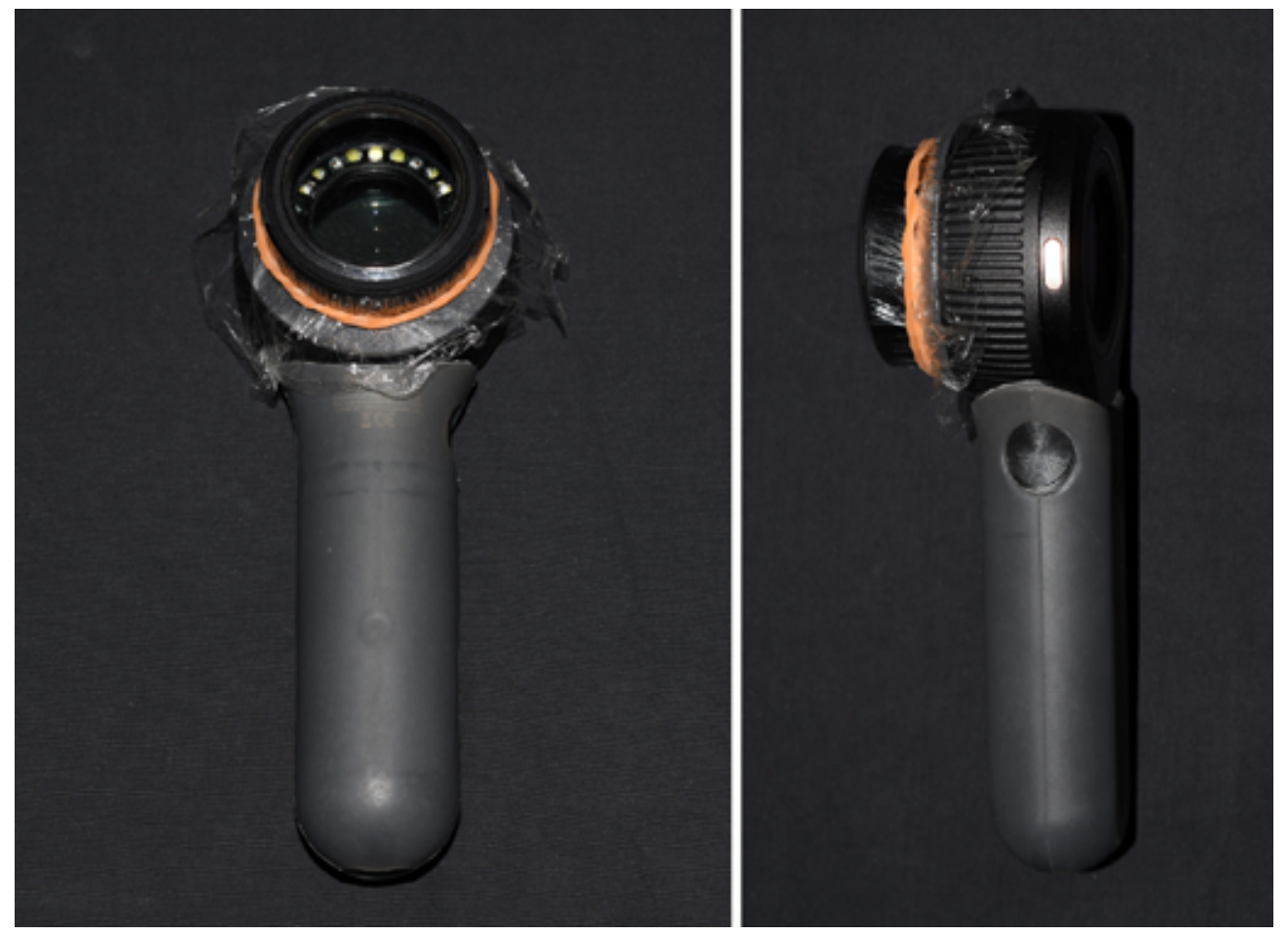

Figure 2. The plastic wrap is firmly stretched over the dermatoscope faceplate then fixed in place with the rubber band.
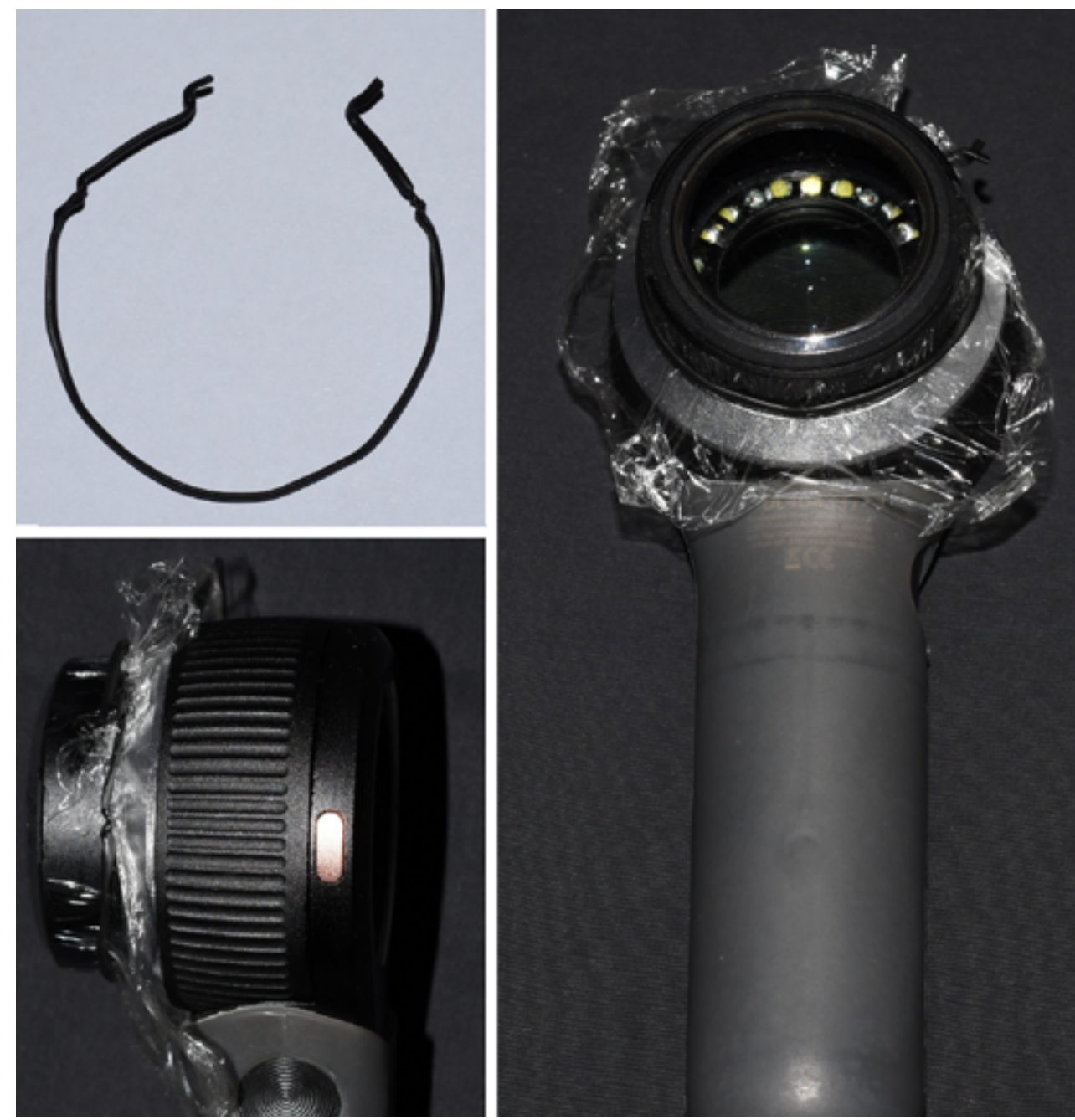

Figure 3. The plastic wrap can also be fixed in place using a metal cable tie. 


\section{References}

1. Mun JH, Park SM, Ko HC, Kim BS, Kim MB. Prevention of possible cross-infection among patients by dermoscopy: a brief review of the literature and our suggestion. Dermatol Pract Concept. 2013;3(4):33-34. DOI: 10.5826/dpc.0304a07. PMID:24282661.
2. Zampino MR, Borghi A, Caselli E, et al. Virologic safety of polyvinyl chloride film in dermoscopic analysis of mucosal areas. Arch Dermatol. 2007;143(7):945-946. DOI: 10.1001/ archderm.143.7.945. PMID:17638745. 
